\title{
A Retrospective Descriptive Study of Stat TPN Orders in the Neonatal Intensive Care Unit
} Lan Dang, M.D. ${ }^{1}$, Chris Durham, Pharm.D. ${ }^{2}$, Carolyn R. Ahlers-Schmidt, Ph.D. ${ }^{1}$, Alissa Rankin, M.P.H. ${ }^{1}$, Jared Shaw ${ }^{2}$, Barry T. Bloom, M.D. ${ }^{1}$

${ }^{1}$ University of Kansas School of Medicine-Wichita, Department of Pediatrics

${ }^{2}$ Wesley Medical Center, Wichita, KS

\begin{abstract}
Background. Total parenteral nutrition (TPN) is used in the Neonatal Intensive Care Unit (NICU) to meet metabolic demand and provide growth. To prevent harm from critical laboratory abnormalities, stat TPNs can be ordered urgently to change the content of infusing TPN. Each stat order breaks the daily cycle and often leads to additional stat orders. Limited supplies of ingredients brought focus on our liberal stat TPN policy and how to reduce the number of stat TPNs safely. The purpose of this project was to evaluate biochemical abnormalities associated with stat TPNs and identify leverage points to reduce stat TPNs in NICU patients.

Methods. Data from 1/1/10 to 6/30/10 were abstracted from Meditech, NeoData, and patient charts for NICU stat TPN orders. Demographics, laboratory results (sodium, potassium, calcium, and glucose), and key variables were gathered and critical laboratory values were identified.

Results. A total of 112 patients had evaluable orders for 255 stat TPNs. Mean gestation was 31 weeks (SD = 5) and birth weight was $1.744 \mathrm{~kg}(\mathrm{SD}=0.993)$. Seven $(3 \%)$ were never infused. Twenty $(12.6 \%)$ of first stat TPNs were from patients taking nothing by mouth. Eighty-eight of first stat TPNs had no critical labs (55\% of initial stat TPNs). Of follow-up stat orders, 43\% (38/89) followed unnecessary initial stat TPNs. Of the 55 abnormalities that generated the initial stat TPNs, 44 (80\%) corrected.

Conclusions. Fifty-two percent of stat TPNs could not be justified. For situations that were justified, $20 \%$ of laboratory abnormalities from initial stat TPNs were not corrected. These data provide an opportunity to reduce unnecessary costs and save limited resources.
\end{abstract}

KS J Med 2012; 5(3):79-82.

\section{Introduction}

Total parenteral nutrition (TPN) is the intravenous infusion of all nutrients necessary to meet metabolic demand and provide growth. ${ }^{1}$ It is used commonly in the Neonatal Intensive Care Unit (NICU) and has shortened hospital stays and improved outcomes of neonates over the past four decades. $^{2}$ Because TPN bypasses enteral filtration and regulation, patients on TPN require close monitoring of laboratory data ${ }^{1}$ to prevent complications such as seizures, apnea, poor tone, and fatal arrhythmias. ${ }^{3}$

Electrolyte abnormalities can be corrected with manipulations to the content of the TPN, changing the sources of fluids or by changing the rate of delivery of the existing TPN. Changes in the content of TPN usually are accomplished through the writing of a daily order, however, many NICUs have the option to use a stat TPN order. To date, there have been no studies on the factors driving stat TPN orders.

A new TPN order is written during morning rounds in our hospital. The order is prepared in the afternoon and hung for infusion around 1800. If the content of the TPN needs to be changed later in the day based upon a change in a patient's nutritional requirements, concerns for necrotizing enterocolitis, or electrolyte 
abnormalities, a new TPN is written and labeled as stat. When a stat TPN is ordered, an additional TPN needs to be ordered later in the day to provide no gap in TPN coverage. Laboratory tests are repeated within 12 hours on infants with stat TPN orders to confirm correction of laboratory abnormalities or to identify additional required actions.

Opportunities for errors arise with each new stat TPN order. More than thirty steps with numerous safety checks are required to make each bag of TPN. In addition, it takes the time of two nurses and over 20 steps to hang each bag safely. Mistakes in any of the steps could lead to patient harm. Chances for infection are encountered with each stat TPN as the line needs to be broken and accessed to administer each new TPN. At our institution, the materials and labor costs associated with each stat TPN order is approximately $\$ 90$.

Along with increased costs and risks associated with each stat TPN order, a shortage of key ingredients, such as cysteine and trace minerals, brought focus on our current ordering process. Our goal was to determine if and how we safely could reduce the number of stat TPN orders.

\section{Methods}

A list of all NICU stat TPN orders was obtained between January 1, 2010 and June 30, 2010 at Wesley Medical Center using Meditech, the electronic medical information system. Information was included about the composition of the existing TPN and/or enteral feeds, the composition of the stat TPN, the time the order was written, the time the TPN was hung, and the times laboratory data were drawn pre- and post-stat TPN order. Laboratory data most proximate to (both before and after) each stat TPN order were obtained. Demographic information also was collected from NeoData, the NICU medical records system. TPN infusion rates were verified by reviewing order sheets.

The first and follow-up stat TPNs were assessed separately. Subsequent stat TPNs were identified as any stat TPNs ordered within 48 hours of a preceding stat TPN. To classify abnormal laboratory data, two different cutoffs were used, "abnormal" as defined by the laboratory reporting system and "critical" as defined by the thresholds used in a recent clinical trial for serious adverse events. ${ }^{4}$ (See Table 1 ).

Table 1. Cutoffs for laboratory data.

\begin{tabular}{|c|c|c|c|c|}
\hline $\begin{array}{c}\text { Laboratory } \\
\text { Variable }\end{array}$ & $\begin{array}{c}\text { Critical Low } \\
\text { Values }\end{array}$ & Low Values & High Values & $\begin{array}{c}\text { Critical High } \\
\text { Values }\end{array}$ \\
\hline Sodium (mmol/L) & 130 & 135 & 148 & 150 \\
\hline $\begin{array}{c}\text { Potassium } \\
\text { (mmol/L) }\end{array}$ & 3.0 & 3.5 & 6.0 & 6.5 \\
\hline $\begin{array}{c}\text { Ionized Calcium } \\
(\mathrm{mg} / \mathrm{dl})\end{array}$ & 4.0 & 4.5 & 5.3 & 6.5 \\
\hline Glucose (mg/dl) & 45 & 60 & 125 & 175 \\
\hline
\end{tabular}




\section{Results}

There were 112 patients who had stat TPNs written from January 1, 2010 to June 30,2010 . The mean birth weight of this population was $1.744 \mathrm{~kg}(+/-0.993)$ and the mean gestational age at birth was 31 weeks (+/- 5). Sixty (54\%) were males, $8(7 \%)$ had necrotizing enterocolitis, and $14(13 \%)$ had intraventricular hemorrhage. There were 259 total stat TPNs during the study period. Four stat TPNs were unevaluable because of insufficient data. Seven stat TPNs were ordered but never hung.

First Stat TPNs. Of the remaining 248 stat TPNs, 159 first stat TPNs were identified. Twenty (13\%) were for patients whose enteral feeds were stopped urgently and were taking nothing by mouth (i.e., NPO). Fifteen patients (9\%) had no associated abnormal laboratory values, 73 (46\%) had abnormal laboratory values that were not critical, and $51(32 \%)$ had critical lab values. Of the 124 first stat TPNs with abnormal or critical laboratory values, 76 (61\%) had one, 32 (26\%) had two, 14 (11\%) had three, and two (2\%) had four. Of the 190 laboratory abnormalities, 55 (29\%) were considered critical; an abnormality in glucose was the most frequent (40\%).

Subsequent Stat TPNs. There were 89 subsequent stat TPNs. Forty-five (51\%) followed stat TPNs with critical laboratory abnormalities and six (7\%) were from patients that went NPO. Thirty-eight (43\%) of these were written as follow-ups to stat TPNs that had non-critical or no laboratory abnormalities.

Impact of First Stat TPN. Pre- and postlaboratory data were compared to see whether the hanging of the stat TPN corrected the abnormality in the desired direction (see Table 2). Correction of critical labs with first stat TPNs failed to occur $20 \%$ of the time; hypo- and hypernatremia were most likely to fail to correct (36.4\% non-correcting).

Table 2. Result of administering first stat TPN.

\begin{tabular}{|c|c|c|c|c|c|}
\cline { 3 - 6 } \multicolumn{2}{c|}{} & Sodium & Potassium & Ion Calcium & Glucose \\
\hline $\begin{array}{c}\text { Critical Low } \\
\text { Values }\end{array}$ & Correcting & 8 & 2 & 1 & 19 \\
\cline { 2 - 6 } & Total & 13 & 2 & 1 & 20 \\
\hline $\begin{array}{c}\text { Critical High } \\
\text { Values }\end{array}$ & Correcting & 6 & 4 & 2 & 2 \\
\cline { 2 - 6 } & Total & 9 & 4 & 3 & 3 \\
\hline
\end{tabular}

\section{Discussion}

A liberal stat TPN policy leads to excessive costs, overuse of limited resources, and increases risks to patients. In this population, more than $43 \%$ of stat TPN orders were written because of abnormal but not critical laboratory values. In this scenario, other methods of correction, such as changing the rate of TPN or waiting to see if the abnormality would self-correct, could have been utilized. An additional 6\% of all evaluable stat TPNs had no laboratory abnormalities and $3 \%$ of stat TPNs were written and made, but never hung. Therefore, more than half (133 of 255) of all evaluable stat TPN orders were not justified by patient condition or critical laboratory.

Even when stat TPNs were justified, there was room for improvement in the management of electrolyte and glucose abnormalities. Twenty percent of stat TPN orders failed to produce desired changes to critical laboratory data. Serum calcium and potassium were most likely and serum sodium was least likely to be corrected by 
changes in TPN composition. This area should be studied further to find more effective ways to correct these biochemical abnormalities.

There were limitations to this retrospective study. Variables which may have influenced each stat TPN may not have been recorded or identified. Also, the percentages of abnormal laboratory values that would have self-corrected or been correctable using interventions other than stat TPN are not known. The flipside is that percentages of these abnormalities would

\section{References}

${ }^{1}$ Thomas DR. Total parenteral nutrition. The Merck Manuals Online Medical Libraries: Nutritional Disorders. April 2009. Available at: http://www.merck. com $/ \mathrm{mmpe} / \mathrm{sec} 01 / \mathrm{ch} 003 / \mathrm{ch} 003 \mathrm{c} . \mathrm{html}$.

2 Pinchasik D. From TPN to breast feedingfeeding the premature infant-2000: Part I. Parenteral nutrition. Am J Perinatol 2001; 18(2):59-72. PMID: 11383702.

${ }^{3}$ Kliegman RM, Jenson HB, Behrman RE. Nelson Textbook of Pediatrics. $17^{\text {th }}$ edition. Philadelphia, Pa: WB Saunders, 2004. ISBN: 0721695566. have become critical without stat TPN also is not known.

No guidelines exist on when laboratory abnormalities warrant a stat TPN order. The implementation of a guideline in our environment for stat TPN orders would decrease the number of unnecessary first stat TPNs and their unnecessary subsequent stat TPNs. These guidelines could offer clinicians alternative methods for correcting subcritical laboratory abnormalities, which potentially could decrease costs and risks to our patient population.

${ }^{4}$ Bloom B, Schelonka R, Kueser T, et al. Multicenter study to assess safety and efficacy of INH-A21, a donor-selected human staphylococcal immunoglobulin, for prevention of nosocomial infections in very low birth weight infants. Pediatr Infect Dis J 2005; 24(10):858-866. PMID: 16220082.

Keywords: neonates, total parenteral nutrition, neonatal intensive care, electrolyte balance 